\title{
Quantitative Visualization of Heat Transfer in Oscillatory and Pulsatile Flows
}

\author{
Cila Herman \\ Department of Mechanical Engineering, The Johns Hopkins University
}

USA

\section{Introduction}

Oscillatory and pulsatile flows arise in a variety of engineering applications as well as in nature. Typical examples include blood flow, breathing, flow in some pipe systems, acoustic systems, etc. Typical engineering applications include enhancement of heat transfer and species transport, chemical species separation, flow control, flow velocity measurement equipment calibration and biomedical applications. Very often these flows are accompanied by heat or mass transfer processes. During the past four decades numerous studies have addressed issues specific for purely oscillatory and modulated or pulsatile flows (oscillatory flow superimposed on a mean steady flow). Recent advances in the study of oscillatory flows were reviewed by Cooper et al. (1993) and Herman (2000). A better understanding of these flows and the accompanying heat transfer processes is essential for the proper design of equipment for such processes and physical situations. Both the experimental study and the computational modeling of oscillatory and pulsatile flows pose specific challenges, which will be addressed in this chapter, with the emphasis on quantitative experimental visualization using holographic interferometry.

Experimental visualization of oscillatory and pulsatile flows and heat transfer requires noninvasive measurement techniques, to avoid affecting the investigated process. In this chapter we discuss holographic interferometry $(\mathrm{HI})$ as a powerful tool in the quantitative visualization of oscillatory and pulsatile flows and heat transfer. Two situations will be considered to demonstrate the applications of the method: (i) the study of self-sustained oscillatory flows and the accompanying heat transfer in grooved and communicating channels and the study of (ii) oscillatory flow and heat transfer in the stack region of thermoacoustic refrigerators.

In this chapter we introduce holographic interferometry as an experimental technique that simultaneously renders quantitative flow and heat transfer data. We demonstrate that for a certain class of problems $\mathrm{HI}$ is superior to conventional flow visualization techniques, such as tracer methods or dye injection, since it can provide not only qualitative but also quantitative insight into certain types of unsteady flows and it does not require the seeding of the flow. Several types of flows and heat transfer processes amenable for quantitative evaluation will be analyzed in the paper. We begin the discussion by introducing the experimental apparatus and technique, followed by the description of the investigated 
physical situation. Next, we present, based on three case studies, visualized temperature fields along with numerous examples of how quantitative data can be extracted from interferometric visualization images. Data reduction procedures, image processing tools, experimental uncertainties as well as advantages and limitations of the method are explained.

\section{Real-time holographic interferometry for quantitative visualization of fluid flow and heat transfer}

Holographic Interferometry (HI) is a well-established measurement and visualization technique widely used in engineering sciences (Vest, 1979; Hauf and Grigull, 1970). In transparent fluids it visualizes refractive index fields, which are related to fluid properties, such as temperature, pressure, species concentration, as well as density in compressible flows. Optical measurement techniques have virtually no "inertia", therefore they are ideal tools for investigation of high-speed, unsteady processes. The combination of HI and highspeed cinematography (that allows high spatial resolutions) is used in the present study to visualize oscillatory or pulsatile flows.

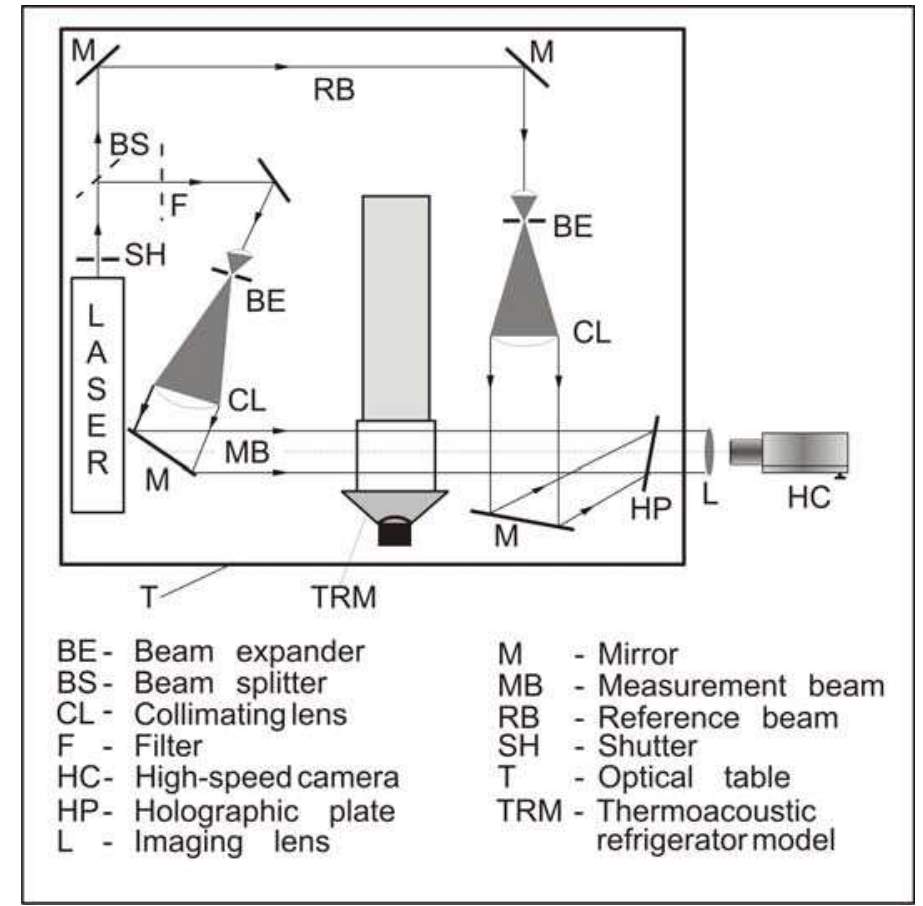

Fig. 1. Optical arrangement for holographic interferometry

\subsection{Optical arrangement for holographic interferometry}

Holographic interferometry uses light as information carrier to provide both qualitative (visual) insight and quantitative data on the investigated physical process. In convective 
heat transfer measurements the temperature fields in the thermal boundary layer above the heated surface and in the transparent working fluid are of particular interest. For more details about the technique, the reader is referred to the comprehensive literature on this topic. General information can be found in the publications such as those of Vest (1979) and Mayinger (1994). Specific information on the optical setup used in our studies can be found in the descriptions by Amon et al. (1992).

A standard optical arrangement for $\mathrm{HI}$ is presented in Fig. 1. The light source is a laser. In our research we used both a $25 \mathrm{~mW}$ Helium-Neon and a $1 \mathrm{~W}$ Argon-Ion laser. The laser power required for analyzing a particular physical process depends on the speed of the process, i.e. the highest frequency of oscillations in oscillatory and pulsatile flows, as well as the sensitivity of the film or digital sensor used to record the high-speed image sequence, in order to be able to resolve the smallest time scales of interest. The type and wavelength of the laser determine the choice of holographic and film materials for highest sensitivity, resolution and contrast, which is especially critical in high-speed applications.

For imaging by $\mathrm{HI}$, the laser beam is divided into a reference beam, RB, and an object beam, $\mathrm{OB}$, by means of a, usually variable, semitransparent mirror (beam splitter), BS, as shown in Fig. 1. Both beams are then expanded into parallel light bundles by a beam expander, BE, which consists of a microscope objective, a spatial filter and a collimating lens. The object beam passes through the test section, TS, with the phase object (representing the refractive index field to be visualized and related to temperature, concentration or density in the evaluation phase) and then falls on the holographic plate, $\mathrm{H}$. The reference beam falls directly onto the holographic plate. The photograph of the optical arrangement for holographic interferometry in the Heat Transfer Lab of the Johns Hopkins University with the thermoacoustic refrigerator model mounted on the optical table is displayed in Fig. 2.

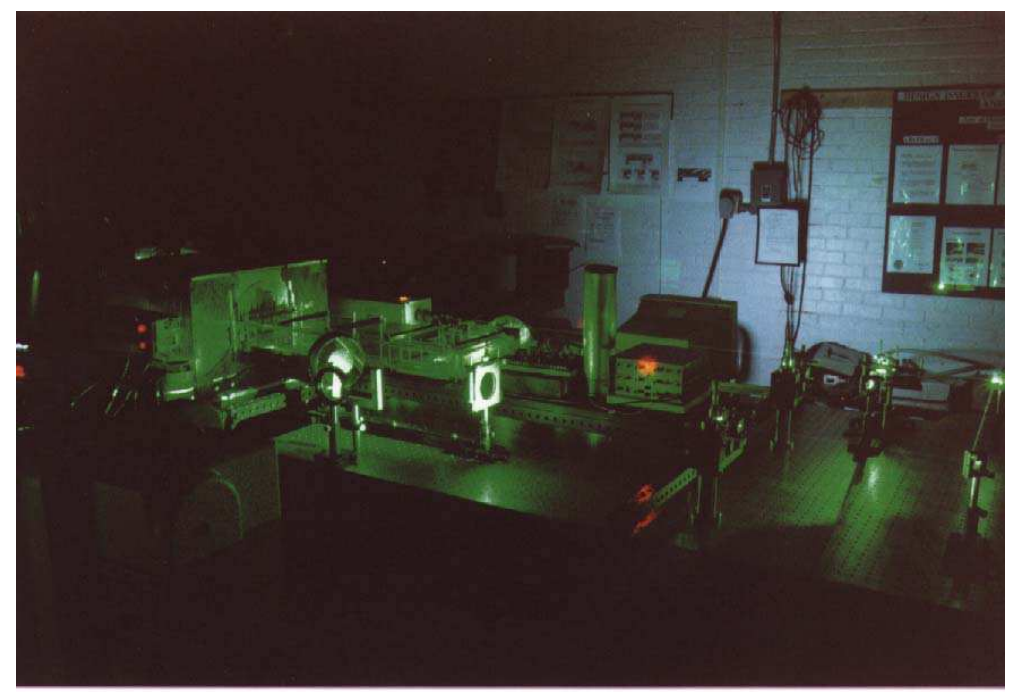

Fig. 2. Photograph of the optical arrangement for holographic interferometry at the Heat Transfer Lab of the Johns Hopkins University and the thermoacoustic refrigerator model mounted on the optical table. 


\subsection{Visualization of temperature fields: infinite and finite fringe field arrangements}

$\mathrm{HI}$ allows the visualization and analysis of high-speed, transient phenomena by using the real-time method, which is a single exposure technique. The visualization is carried out in two steps. First, the reference state (usually with the fluid in the measurement volume at ambient temperature) is recorded on the holographic plate. Next, the holographic plate is developed, bleached, dried and exactly repositioned into a precision plate holder. In the second step, the reference state of the object under investigation is reconstructed by illuminating the holographic plate with the reference beam. At the same time, the investigated physical process is initiated (in our experiment the blocks in the wind tunnel are heated or the thermoacoustic refrigerator is activated). The heating causes the refractive index of the fluid in the measurement volume to change, and, consequently, this causes the object wave to experience a phase shift on its way through the test section. The difference between the reference state recorded earlier and the new state of the fluid in the measurement volume, i.e. the phase shift between reference and measurement beams, is visualized in the form of a macroscopic interference fringe pattern. This fringe pattern can be recorded with a photographic camera or a high-speed camera (when the process is unsteady).

If, during the measurement, the object wave is identical to the original state for which the reference hologram was recorded (the object is unheated, for example), no interference fringes will appear. This state can be adjusted before initiating the experiment, and the corresponding method of reconstruction is called the infinite fringe field alignment. The infinite fringe field alignment was used in all measurements reported in this paper. When the heat transfer process is initiated, the object wave passing through the test section becomes distorted, and behind the hologram the object and reference waves interact to form a macroscopic interference pattern. In our study we record this fringe pattern with a highspeed camera with speeds of up to 10,000 image frames per second. It is desirable to record around 10 images or more during one period of oscillations to achieve good reconstruction accuracy. The interferometric fringes obtained using the infinite fringe field alignment correspond to isotherms, and are suitable, apart from the fairly common temperature measurements, also for the quantitative visualization of fluid flow phenomena, which will be demonstrated in this chapter.

Another alignment of the optical equipment frequently used in interferometric measurements is the finite fringe field alignment. In this method a small tilt is applied to the mirror $\mathrm{M}$ in Fig. 1 that projects the reference beam onto the holographic plate. At ambient conditions this tilt will cause a regular, parallel macroscopic fringe pattern to form in the field of view. Our experience indicates that the finite fringe field alignment is less suitable for quantitative flow visualization, since the fringe patterns cannot be easily and intuitively related to the flow field. The finite fringe field alignment is frequently used when temperature gradients on the heated surface are measured (rather than temperatures). An example contrasting images obtained by the infinite and finite fringe field alignments is shown in Fig. 3. Both interferometric images visualize temperature fields around two heated stack plates in crossflow. The thermal boundary layers can be identified in both alignments, the fringes in the infinite fringe field alignment visualize the isotherms in the thermal boundary layer.

In heat transfer measurements, that were the original and primary objective of our investigations, high spatial resolutions are required to analyze the thin thermal boundary 
layers in the vicinity of the heated surface in forced convection, such as those shown in the bottom image of Fig. 3. In order to achieve sufficient accuracy in heat transfer measurements, the interferometric images in our experiments were recorded on $16 \mathrm{~mm}$ high-speed film first, then scanned, digitized with resolutions up to $2700 \mathrm{dpi}$, and finally evaluated quantitatively using digital image processing techniques. The cost of video equipment suitable for these high-speed heat transfer measurements would have been prohibitive in addition to unsatisfactory spatial resolution, since the resolution of images recorded by digital video cameras decreases with increasing recording speed. Depending on the thickness of the thermal boundary layer and the refractive index of the working fluid, tens to hundreds of fringes may need to be resolved accurately over a distance of few millimeters. In flow visualization experiments the spatial resolution is less critical than in temperature measurements, since the fringes relevant for the characterization of flow phenomena in the main channel and recirculating regions are wider than in the thermal boundary layer.

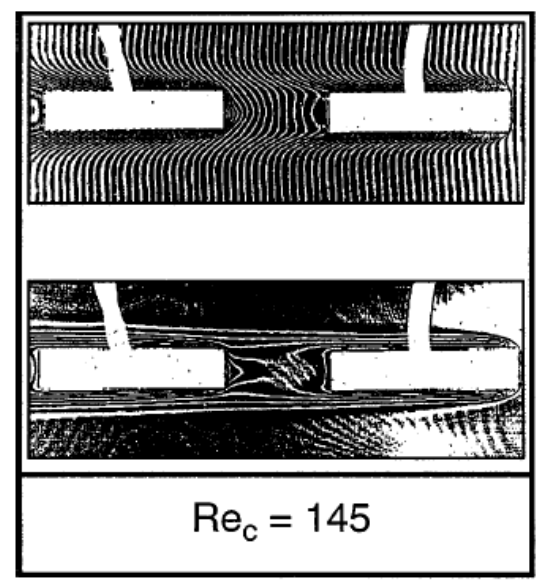

Fig. 3. Temperature fields around two heated plates in crossflow in a rectangular channel visualized by HI using the finite fringe field arrangement (top) and the infinite fringe field arrangement (bottom).

\section{Physical situations}

Oscillating flows can be classified according to the method used to generate the oscillations, flow geometry, role of compressibility, character of the undisturbed flow as well as other flow parameters that will influence the development of the flow field and the heat transfer process. The impact of self-sustained oscillation in grooved and communicating channels as well as the impact of acoustic oscillations on convective heat transfer and the role of compressibility in the stack of a thermoacoustic refrigerator will be addressed in this paper.

\subsection{Self-sustained oscillatory flows in grooved and communicating channels}

Self-sustained oscillatory flows in grooved and communicating channels were visualized in wind tunnels specially designed to allow accurate measurements by $\mathrm{HI}$ using air as the 
working fluid. The length of the path of light across the heated region is a critical design parameter that determines the number of fringes present in the interferometric image for a prescribed temperature difference.

During the past four decades the development of compact heat transfer surfaces has received considerable attention in the research community. It was found that oscillation of the driving flow is a promising approach to heat transfer augmentation (Ghaddar et al. 1986a and 1986b). Resonant heat transfer enhancement is a passive heat transfer enhancement technique, which is appropriate for systems with naturally occurring separated flows, such as the grooved and communicating channels shown in Fig. 4 . Grooved channels are typically encountered in electronic cooling applications (the heated blocks represent electronic chips mounted on a printed circuit board) and communicating channels represent a model of the rectangular plate fin, offset-fin and offset strip-fin flow passages of compact heat exchangers as well as heat sinks used in electronic packaging solutions.

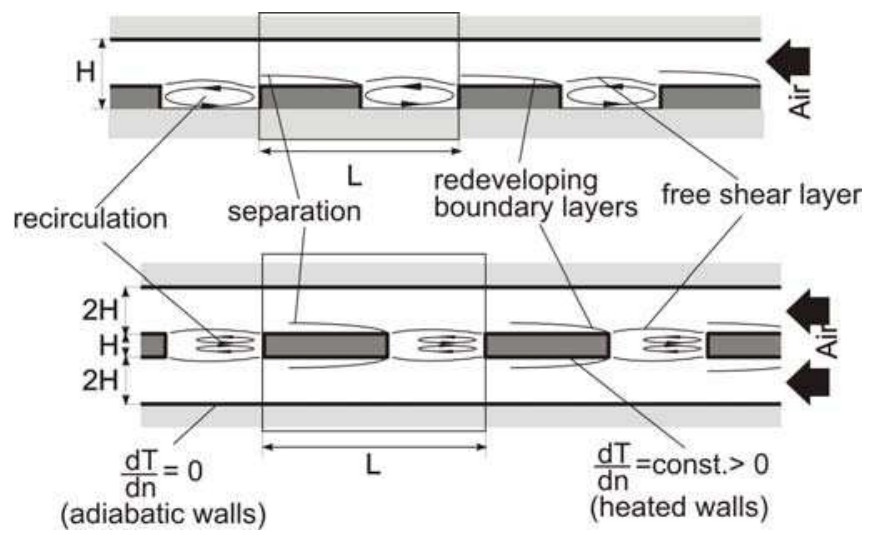

Fig. 4. Schematic of the geometries and physical situations for the study of self-sustained oscillatory flows in grooved (top) and communicating (bottom) channels

The enhanced surfaces we investigated involve the repeated formation and destruction of thin thermal boundary layers by interrupting the heat transfer surface in the streamwise direction, as shown in the schematic in Fig. 4. In addition to their practical significance, the two situations presented in Fig. 4 are examples of separated shear flows featuring complex interactions between separated vortices, free shear layers and wall bounded shear layers. In both channel geometries, two main flow regions can be identified: (i) the bulk flow in the main channel and (ii) the weak recirculating vortex flow in the groove or communicating region. They are separated by a free shear layer. In laminar, steady-state conditions there is virtually no exchange of fluid between these two regions. The results of Patera and Mikic (1986), Karniadakis et al. (1987), Greiner et al. (1990) and Greiner (1991) showed that selfsustained oscillations develop in such flow configurations at a relatively low Reynolds number in the transitional regime, and the interaction of separated flow with imposed unsteadiness leads to lateral convective motions that result in overall transport enhancement. 
The heated blocks attached to the bottom of the grooved channel and the plates in the central plane of the communicating channels were heated electrically. The thermal boundary conditions on the surface of the heated blocks are described by constant heat flux, as indicated in Fig. 4. The top and bottom plane walls of the test section are manufactured of low thermal conductivity material to maintain approximately adiabatic thermal boundary conditions. Details on the experimental setup and instrumentation are available elsewhere (Amon et al. 1992; Farhanieh et al., 1993; Kang, 2002).

In both channels, above a critical Reynolds number and at sufficient downstream distance, a periodically, fully developed flow regime is established. This was the region of interest in our visualization experiments, since the instantaneous velocity and temperature fields repeat periodically in space. Therefore temperature fields were visualized in the region of the ninth heated block (there were 11 blocks in the test section), sufficiently far downstream from the channel entrance to satisfy the periodicity requirement. The channel height to spanwise dimension aspect ratio is selected to ensure that the flow and temperature fields investigated by $\mathrm{HI}$ are two-dimensional.

\subsection{Oscillatory flow in a thermoacoustic refrigerator}

$\mathrm{HI}$ can also be applied to visualize time dependent temperature distributions in oscillating flows with zero mean velocity. A need for such measurements arose in the investigations of heat transfer in thermoacoustic refrigerators. Thermoacoustic refrigeration is a new, environmentally safe refrigeration technique that was developed during the past two decades (Wheatley et al. 1983; Swift 1988). The schematic of a thermoacoustic refrigerator is presented in Fig. 5. The purpose of the acoustic driver is to generate an acoustic standing wave in the resonance tube. Thus, the working fluid in the resonance tube oscillates with zero mean velocity.

Over the past decades environmental concerns have become increasingly important in the design and development of energy conversion and refrigeration systems. Thermoacoustic energy conversion was introduced into engineering systems during the past four decades as a new, alternative, environmentally safe energy conversion technology. It uses noble gases and mixtures of noble gases as working fluids rather than hazardous refrigerants required for the vapor compression cycle. A thermoacoustic system can operate both as a prime mover/engine, when a temperature gradient and heat flow imposed across the stack leads to the generation of acoustic work/sound in the resonator. When reversing the thermodynamic cycle, the thermoacoustic system functions as a refrigerator: acoustic work is used to pump heat from the low temperature reservoir to release it into a higher temperature ambient. Heat transfer in the stack region of the thermoacoustic refrigerator was the focus of our visualization experiments.

The schematic of a half-wavelength thermoacoustic refrigerator is shown in Fig. 5. Energy transport in thermoacoustic systems is based on the thermoacoustic effect. Using an acoustic driver, the working fluid in the resonance tube is excited to generate an acoustic standing wave. When introducing a stack of plates of length $\Delta \mathrm{x}$ at a location specified by $\mathrm{x}_{\mathrm{c}}$ into the acoustic field, a temperature difference $\Delta \mathrm{T}$ develops along the stack plates. This temperature difference is caused by the thermoacoustic effect. The thermoacoustic effect is visualized in our study using high-speed holographic interferometry. In HI both temperature and pressure variations impact the refractive index and they are both present in our 
thermoacoustic system. Therefore, temperature variations need to be uncoupled from pressure variations in our evaluation process, to accurately quantitatively visualize the oscillating temperature fields around the stack plate.

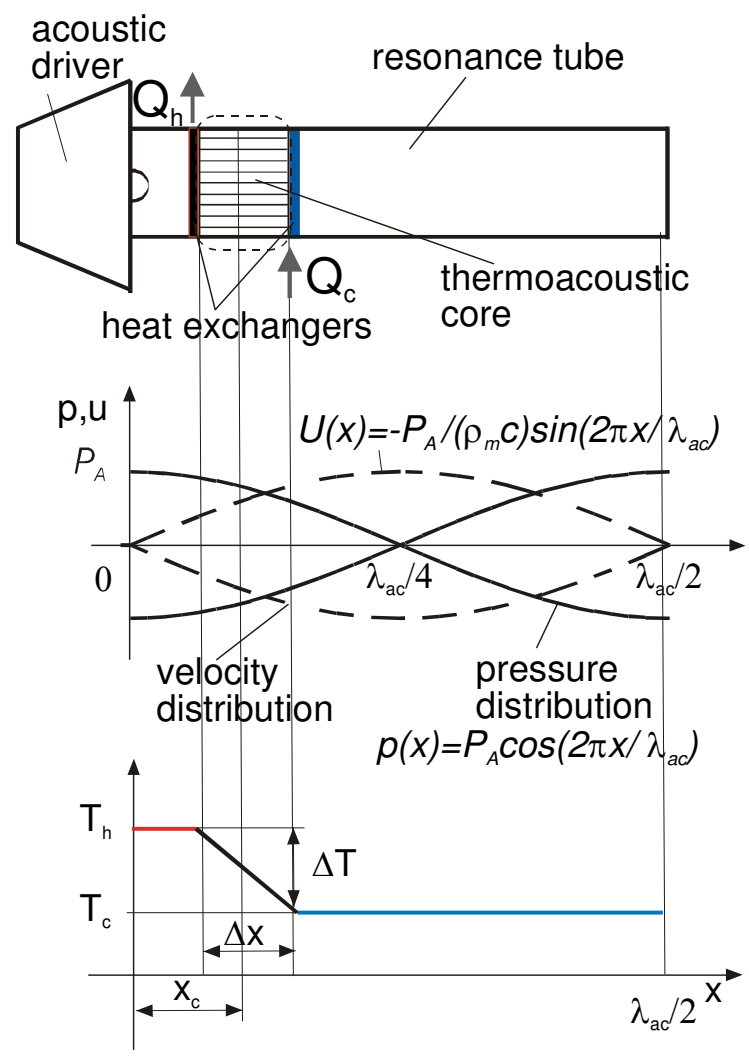

Fig. 5. Schematic of the thermoacoustic refrigerator, pressure and velocity distributions in the resonance tube and temperature distribution along the stack and in the resonator.

In the top portion of Fig. 5, a schematic of a thermoacoustic refrigerator is shown. The length of the resonance tube in the study corresponds to half the wavelength of the acoustic standing wave, $\lambda_{a d} / 2$. The corresponding pressure and velocity distributions are displayed in the middle image in Fig. 5. A densely spaced stack of plates of length $\Delta x$ is introduced at a location specified by the stack center position $x_{c}$ into the acoustic field. During the operation of the refrigerator a temperature difference $\Delta T$ develops along the stack plates (bottom image in Fig. 5). By attaching heat exchangers on the cold and hot ends of the stack, heat $Q_{c}$ can be removed from a low temperature reservoir, pumped along the stack plate to be delivered into the high temperature heat exchanger and ambient as $Q_{h}$. The temperature difference forming along the stack is caused by the thermoacoustic effect. This paper focuses on the visualization of the oscillating temperature fields in the thermoacoustic stack near the edge of the stack plates, which allows the visualization of the thermoacoustic effect. 
The mechanism of thermoacoustic heat pumping (Swift, 1988) is illustrated in the schematic in Fig. 6, by considering the oscillation of a single gas parcel of the working fluid along a stack plate. The gas parcel begins the cycle at a temperature $T$. In the first step, the gas parcel moves to the left, towards the pressure antinode, the movement caused by the acoustic wave. During this displacement it experiences adiabatic compression, which causes its temperature to rise by two arbitrary units to $T^{++}$(Step 1 in Fig. 6). In this state the gas parcel is warmer than the stack plate and irreversible heat transfer from the parcel towards the stack plate takes place (Step 2 in Fig. 6). The resulting temperature of the gas parcel after this heat loss step is $T^{+}$. On its way back to the initial location the gas parcel experiences adiabatic expansion and cools down by two arbitrary units, to the temperature $T^{-}$(Step 3 in Fig. 6). At this state the gas parcel is colder than the stack plate and irreversible heat transfer from the stack plate towards the gas parcel takes place (Step 4 in Fig. 6). After these four steps the gas parcel has completed one thermodynamic cycle and reached its initial location and temperature $T$. At this point the cycle can start again. In this paper we visualize the oscillating temperature distributions near the edge of two stack plates to visualize the thermoacoustic effect.

(1) adiabatic compression

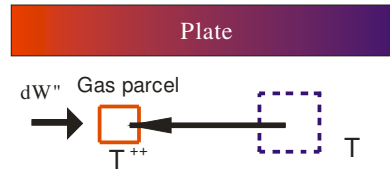

(3) adiabatic expansion

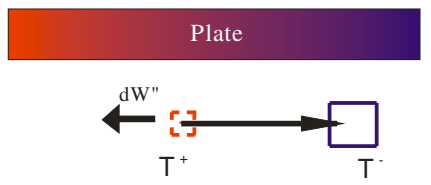

(2) irreversible heat transfer

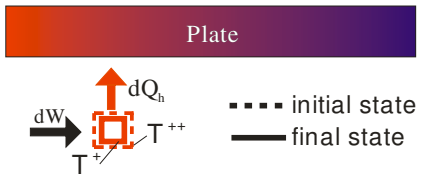

(4) irreversible heat transfer

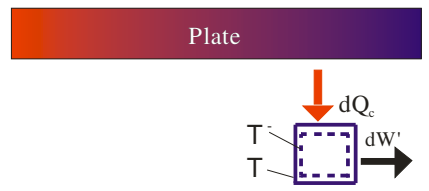

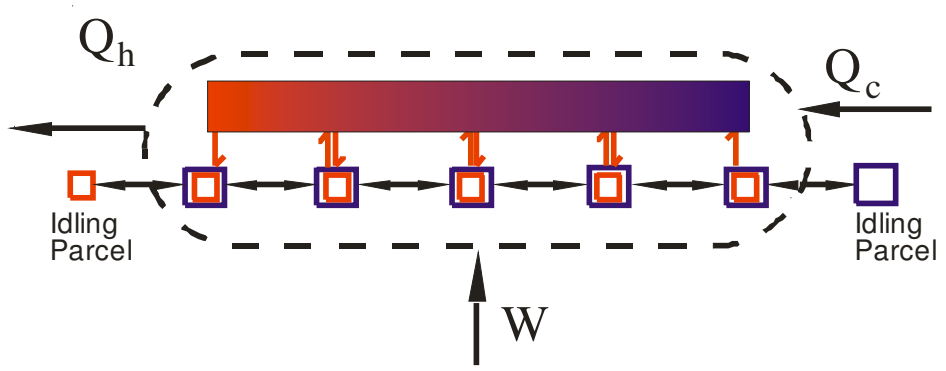

Fig. 6. Heat pumping cycle along a stack plate by considering the oscillation of one gas parcel. The four steps of the cycle are: $1: T \rightarrow T^{++}$adiabatic compression, $2: T^{++} \rightarrow T^{+}$ irreversible heat transfer, 3: $T^{+} \rightarrow T^{-}$adiabatic expansion and $4: T^{-} \rightarrow T$ irreversible heat transfer.

There are many gas parcels subjected to this thermodynamic cycle along each stack plate, and the heat that is delivered to the plate by one gas parcel is transported further by the adjacent parcel, as illustrated in the bottom portion of Fig. 6 . The result of this transport is a 
temperature gradient developing along the stack plates. The described cycle can also be reversed by imposing a temperature gradient $\Delta T$ along the stack plates. In this situation the directions of irreversible heat transfer and work flux are reversed, and the thermoacoustic device operates as a prime mover, also known as the thermoacoustic engine. Thermoacoustic prime movers can be used to generate acoustic work that can drive a thermoacoustic refrigerator, a pulse tube or a Stirling refrigerator. The advantage of this solution is a refrigeration system that does not require moving parts (especially at the low temperature). The combination of $\mathrm{HI}$ and high-speed cinematography (that allows the high spatial resolutions needed in our study) is used to visualize the thermoacoustic effect at the edge of the thermoacoustic stack plates. In the thermoacoustic resonator the temperature fields oscillate with a frequency of $337 \mathrm{~Hz}$. Therefore sampling rates of the order of 5,000 frames per second were needed to accurately resolve the temporal evolution of the physical process. At the same time high spatial resolutions of up to $2700 \mathrm{dpi}$ were realized.

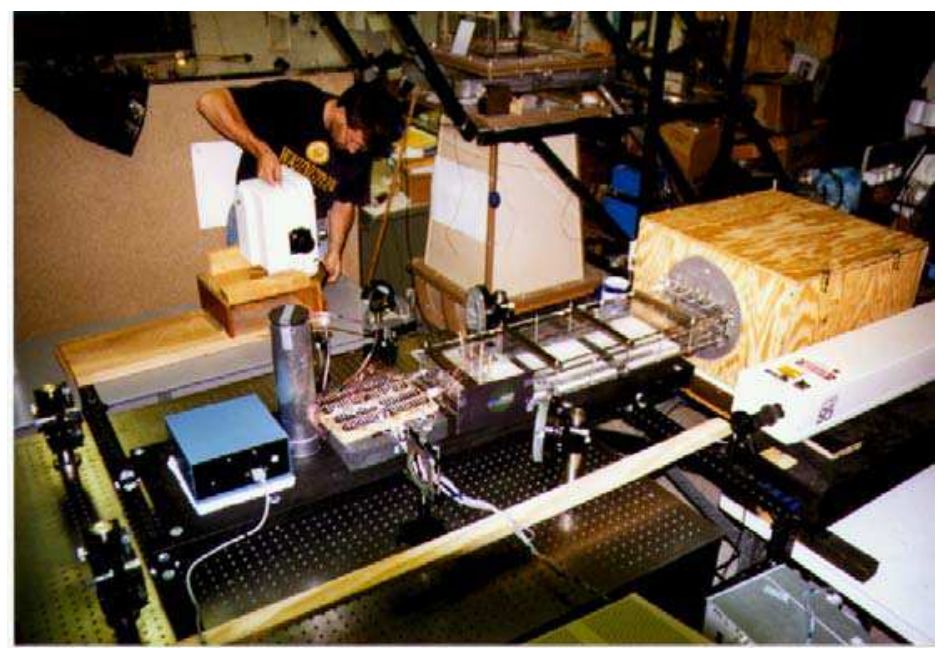

Fig. 7. Thermoacoustic refrigerator model used in the visualization experiments assembled on the optical table in the Heat Transfer Lab of JHU with the components for holographic interferometry.

In Figure 7, the photograph of the thermoacoustic refrigerator model used in the visualization experiments described in this paper is shown. To investigate temperature fields in the region of the stack and heat exchangers using $\mathrm{HI}$, we built a thermoacoustic refrigerator model with a transparent stack region that allows the irradiation of the measurement volume with laser light. The loudspeaker (Electro Voice EVM-10M) was used to generate an acoustic standing wave in the resonance tube. The $337 \mathrm{~Hz}$ input signal for the loudspeaker was generated by an HP 8116A function generator and amplified using a Crown DC-300A Series II amplifier, before being led to the loudspeaker. A dynamic pressure transducer (Sensym SX01) was mounted at the entrance of the resonance tube to measure the acoustic pressures. At this location the transducer measures the dynamic peak pressure amplitude $P_{A}$. The drive ratio in the system is defined as the ratio of peak pressure amplitude $P_{A}$ to the mean pressure $p_{m}$ within the working fluid, $D R \equiv P_{A} / p_{m}$, and it is 
determined from this measurement. Experiments were conducted for drive ratios ranging from $1 \%$ to $3 \%$. The length of the resonance tube $(510 \mathrm{~mm})$ matches half the wavelength of the acoustic standing wave $\lambda_{a c} / 2$. Visualization experiments were carried out on two stack plates. The dimensions of the stack plates are: plate spacing $3 \mathrm{~mm}$, stack length $76 \mathrm{~mm}$ and stack center position $127 \mathrm{~mm}$. To capture details of the movements and to resolve the unsteady temperature fields as a function of time, we used a high-speed film camera, which is capable of recording speeds up to 10,000 picture frames per second. This speed corresponds to a temporal resolution of $0.1 \mathrm{~ms}$.

When applying $\mathrm{HI}$ to the visualization of temperature fields in a thermoacoustic refrigerator, the experimenter faces the problem that the changes in the refractive index cannot be directly related to temperature changes. This is the case because acoustic pressure variations cannot be neglected in the evaluation. Therefore, we developed a new interpretation and evaluation procedure for the interferometric fringe pattern that allows accurate measurements of oscillating temperature fields by accounting for the effect of periodic pressure variations. For a complete description of the unsteady temperature distributions it was also necessary to include frequency and phase measurements into the evaluation procedure.

\section{Reconstruction of temperature fields in the presence of pressure variations}

The feature that makes $\mathrm{HI}$ a powerful measurement tool is the possibility to detect optical path length differences $\Delta \Phi$ between an object wave and a reference wave in the nanometer range. These path length differences $\Delta \Phi$ are the multiple $S$ of the wavelength $\lambda$ of laser light, and they can be visualized in form of interference fringes. The interference fringes can then, in the case of a transparent phase object (Vest, 1979), be related to a difference $\Delta n$ in the refractive index along the optical paths of the object and reference waves as follows

$$
\Delta \Phi=S \cdot \lambda=\Delta n \cdot L .
$$

Equation (1) holds when the refractive index along the direction of transillumination, along the phase object of the optical path length $L$, can be considered constant. In conventional applications of $\mathrm{HI}$ the difference $\Delta n$ in the refractive index is easily related to a single field variable of interest. Such field variables are density or pressure in the case of aerodynamic applications, or temperature and concentration in heat and mass transfer applications of $\mathrm{HI}$, respectively. Most applications of high-speed HI reported in the literature were limited to physical situations in which the difference $\Delta n$ in the refractive index is caused by a single field variable.

In many heat and mass transfer processes, such as chemical reactions, the experimenter is interested in simultaneously measuring temperature $T$ and species concentration $C$. In such a situation the difference $\Delta n(T, C)$ in the refractive index depends on two field variables, temperature as well as concentration, and Equation (1) can be written as (Panknin, 1972)

$$
S \cdot \lambda=L \cdot\left[\left.\frac{\partial n}{\partial T}\right|_{C} \cdot \Delta T+\left.\frac{\partial n}{\partial C}\right|_{T} \cdot \Delta C\right] .
$$


Equation (2) represents a more general form of fringe interpretation in the study of phase objects. The difficulty the experimenter is facing in the quantitative evaluation is that interference fringes cannot be easily interpreted as isotherms or lines of constant concentration. In order to determine both field variables, temperature and concentration, in such a situation, Panknin (1977) took advantage of the fact that the difference $\Delta n$ in the refractive index also depends on the wavelength $\lambda$ of the laser light. Therefore he used two lasers operating at two different wavelengths $\lambda_{i}$ in his experiments, and applied Equation (2) two times to resolve the interference fringes for temperature as well as concentration.

In the case of acoustically driven flow in our thermoacoustic system we are facing a similar situation, since, in addition to the oscillating temperature field, periodic pressure variations are also present in the fluid. Consequently, the difference $\Delta n(T, p)$ in the refractive index depends on both field variables, temperature as well as pressure, and Equation (1) can be written as

$$
S \cdot \lambda=L \cdot\left[\left.\frac{\partial n}{\partial T}\right|_{p} \cdot \Delta T+\left.\frac{\partial n}{\partial p}\right|_{T} \cdot \Delta p\right] .
$$

Again, Equation (3) shows that the interference fringes cannot directly be interpreted as isotherms or isobars. In the paper the impact of periodic pressure variations on the fringe interpretation is discussed first, and an evaluation formula that expands the applicability of $\mathrm{HI}$ to temperature measurements in the presence of pressure variations is introduced. This evaluation formula reduces to its conventional form when the pressure of the measurement state equals the pressure of the reference state. The drive ratio, $D R$, the ratio of the peak pressure amplitude to the mean pressure in the working fluid

$$
D R=\frac{P_{A}}{p_{m}}
$$

is the parameter describing the magnitude of the periodic pressure fluctuations. From Equation (3), it follows that additional information regarding the pressure variations is necessary to be able to resolve the interference fringes for temperature. This information can be derived from the acoustic field. We demonstrated that the interference fringes can be approximated as "quasi"-isotherms because the temperature of one interference fringe can vary, depending on the magnitude of the pressure variations, by up to $18 \mathrm{~K}$ in the present study. We also demonstrate that the conventional evaluation formula can be applied only in cases when the experimenter is interested in time averaged temperature distributions. Furthermore, an analytical error function was derived and applied to correct the unsteady temperature distribution determined with the conventional evaluation formula. The result of this correction shows good agreement with a simple theoretical model.

The reconstruction of temperature fields in the presence of pressure variations involves a series of steps. After expanding the difference in the refractive index $\Delta n$ appearing in Equation (1), we obtain the equation of ideal interferometry (Vest, 1979; Hauf and Grigull, 1970) in the form

$$
S(x, y, t) \cdot \lambda=\left(n(x, y, t)-n_{\infty}\right) \cdot L,
$$

that relates the interference order $S(x, y, t)$ to the refractive index field $n(x, y, t)$ of the measurement state. We note that these parameters are not only functions of the two spatial 
coordinates $x$ and $y$, as in conventional applications of Equation (5), but also time dependent quantities, since we are investigating an unsteady process. The wavelength $\lambda$, the refractive index $n_{\infty}$ of the reference state and the spanwise dimension (optical pathlength) $L$, are known constants, and therefore the refractive index field can be reconstructed from the interference fringe pattern. Equation (5) implies an averaging of the refractive index in the spanwise direction $L$ (along the light beam). Therefore, the experimental setup has been designed to maintain the refractive index along the spanwise direction constant, and thus allow the experimenter to deal with a simplified 2D model of the physical process. To simplify the present discussion, we also neglect refraction of the laser beam and assume that the beam passes along a straight line through the test section. For gases with a refractive index $n \approx 1$, such as air, the Gladstone-Dale equation

$$
\bar{r}(\lambda)=\frac{2}{3} \frac{1}{\rho(x, y, t)}(n(x, y, t)-1)
$$

can be applied to relate the refractive index field $n(x, y, t)$ to the density $\rho(x, y, t)$ of the working fluid. The specific refractivity $\bar{r}(\lambda)$ appearing in Equation (6) is a material specific constant for a given wavelength $\lambda$ of laser light. When comparing the measurement state to the reference state, the Gladstone-Dale equation can be written as

$$
\frac{n(x, y, t)-1}{n_{\infty}-1}=\frac{\rho(x, y, t)}{\rho_{\infty}} .
$$

Substituting Equation (7) into (5) we can relate the density field $\rho(x, y, t)$ of the measurement state to the interference order $S(x, y, t)$ as

$$
\frac{\rho(x, y, t)}{\rho_{\infty}}=\frac{S(x, y, t) \cdot \lambda}{L \cdot\left(n_{\infty}-1\right)}+1 .
$$

The goal of this derivation is to find a relationship between the temperature field $T(x, y, t)$, describing the measurement state, and the interference order $S(x, y, t)$. Therefore, we substitute the ideal gas law in the form

$$
\frac{\rho(x, y, t)}{\rho_{\infty}}=\frac{p(x, y, t)}{p_{\infty}} \cdot \frac{T_{\infty}}{T(x, y, t)},
$$

into Equation (8) to eliminate density and obtain the following evaluation formula

$$
T(x, y, t)=\frac{T_{\infty}}{1+a\left(T_{\infty}\right) \cdot S(x, y, t)} \frac{p(x, y, t)}{p_{\infty}},
$$

describing the temperature field as a function of the interference order $S(x, y, t)$ as well as the pressure $p(x, y, t)$. The evaluation constant $a\left(T_{\infty}\right)$ in Equation (10) is defined as

$$
a\left(T_{\infty}\right) \equiv \frac{\lambda}{L \cdot\left(n_{\infty}-1\right)}=\frac{2}{3} \frac{\lambda}{L} \frac{R \cdot T_{\infty}}{\bar{r}(\lambda) \cdot p_{\infty}} .
$$


Equation (10) incorporates the expansion of the conventional evaluation formula for HI, when the changes in the refractive index are caused not only by temperature changes, but also by pressure variations. Since it has been shown that the temperature field $T(x, y, t)$ under these conditions is a function $f(S, p)$ of interference order $S(x, y, t)$ and pressure $p(x, y, t)$, it is easily understood that the interference fringes cannot be directly related to isotherms. Consequently, in order to apply Equation (10) to quantitatively reconstruct the temperature field $T(x, y, t)$, information on the interference order $S(x, y, t)$ and the measurement state's pressure $p(x, y, t)$ is required. The interference order $S(x, y, t)$ can be determined from the recorded interferometric images, which is not the case for the measurement state's pressure $p(x, y, t)$.

In conventional applications of $\mathrm{HI}$ this complication is avoided by conducting the experiments such, that the pressure $p_{\infty}$ of the reference state equals the pressure $p(x, y, t)$ of the measurement state. Therefore, in such cases the pressure ratio $p(x, y, t) / p_{\infty}$ equals unity, and Equation (10) reduces to the evaluation formula used in conventional applications of $\mathrm{HI}$ in heat transfer measurements (Hauf and Grigull, 1970). The infinite fringe field alignment offers an additional advantage in the application discussed in the present paper: images obtained through these experiments also contain important information about the flow field. Thus, qualitative and quantitative information about the temperature field as well as the flow field is obtained simultaneously.

In the case of an acoustically driven flow it is naturally not possible to maintain the pressure $p(x, y, t)$ of the measurement state constant. Therefore, we acquire the additional information needed to determine the temperature field $T(x, y, t)$, from the acoustic field. For an acoustic field the pressure variations can be expressed as

$$
p(x, y, t)=p_{m}(x, y)+\delta p(x, y, t) .
$$

Substituting Equation (12) into (10) and taking advantage of the fact that the mean pressure $p_{m}$ of the acoustic field in our experiment corresponds to the pressure $p_{\infty}$ of the reference state, we obtain

$$
T(x, y, t)=\frac{T_{\infty}}{1+a\left(T_{\infty}\right) \cdot S(x, y, t)}\left(1+\frac{\delta p(x, y, t)}{p_{m}}\right)
$$

In Equation (13) the term $\delta p(x, y, t) / p_{m}$ describes the periodic pressure fluctuations. For an acoustic field this pressure term is generally at least one to two orders of magnitude smaller than unity (in our measurements $\left[\delta p / p_{m}\right]_{\max }=0.03$ for a drive ratio of $3 \%$ ). Thus, one is tempted to neglect this pressure term and treat the measurement state as having the constant pressure $p_{\infty}$ of the reference state, to arrive at the conventional evaluation formula

$$
T(x, y, t)=\frac{T_{\infty}}{1+a\left(T_{\infty}\right) \cdot S(x, y, t)} .
$$

However, such an approximation may or may not have a significant impact on the temperature measurements, depending on the measurement parameter the experimenter is interested in.

In acoustically driven flow the working fluid is adiabatically compressed and expanded. These compression and expansion cycles cause small temperature fluctuations $\delta T(x, y, t)$ 
around a mean temperature $T_{m}(x, y)$. Consequently, we can describe the temperature field $T(x, y, t)$, similar to the way we have done it with the pressure, as a linear combination of the mean temperature distribution $T_{m}(x, y)$ and a small temperature fluctuation $\delta T(x, y, t)$. Furthermore, we can assume a harmonic time dependence for both temperature and pressure fluctuations, such that the temperature field can be written as

$$
T(x, y, t)=T_{m}(x, y)+T_{A}(x, y) \cdot e^{i \omega t}
$$

and the pressure as

$$
p(x, y, t)=p_{m}+p_{A}(x, y) \cdot e^{i \omega t} .
$$

Next we will apply these considerations to emphasize two important aspects in the application of Equation (14) to temperature measurements in the presence of pressure variations: (i) the impact on fringe interpretation and (ii) the impact on measurements of the temperature field $T(x, y, t)$.

The advantage of Equation (14) when compared to Equation (13) is its simplicity: the temperature field $T(x, y, t)$ is described as a function $f(S)$ of the interference order $S(x, y, t)$ only. Consequently, we can assign a constant temperature $T_{S_{0}}$ to each interference order $S_{0}$, and interpret the interference fringes as isotherms. In order to quantify the influence of pressure variations, we will now focus our attention on the temperature $T_{S_{0}}$ that is assigned to an interference order $S_{0}$ through Equation (14). If we substitute the small pressure fluctuations of Equation (16) into Equation (13) and consider the interference order $S_{0}$ of one interference fringe to be constant, we obtain

$$
T_{S_{0}}(x, y, t)=\frac{T_{\infty}}{1+a\left(T_{\infty}\right) \cdot S_{0}}\left(1+\frac{p_{A}(x, y) e^{i \omega t}}{p_{m}}\right) .
$$

Equation (17) confirms the conclusion that one interference fringe cannot be directly related to an isotherm, because its temperature is a function of the spatially as well as time dependent pressure fluctuation. However, if we average with respect to time over one period of oscillations we obtain

$$
\overline{T_{S_{0}}}=\frac{1}{\tau} \int_{0}^{\tau} \frac{T_{\infty}}{1+a\left(T_{\infty}\right) \cdot S_{0}}\left(1+\frac{p_{A}(x, y) e^{i \omega t}}{p_{m}}\right) d t=\frac{T_{\infty}}{1+a\left(T_{\infty}\right) \cdot S_{0}} .
$$

Equation (18) represents the conventional evaluation formula identical to Equation (14) for one particular interference fringe with the interference order $S_{0}$. Thus, we can conclude that using Equation (14) in the presence of periodic pressure variations, can be interpreted as approximating the temperature of one interference fringe with its time averaged temperature value. Since in this case we are dealing with approximated, time averaged isotherms, we will call them "quasi"-isotherms. To emphasize this fact, let us consider the temperature changes an interference fringe experiences in a typical experiment of the present study. For this purpose let us assume that we can assign a time averaged temperature $\overline{T_{S_{0}}}=300 \mathrm{~K}$ to one particular interference fringe and that the experiment was conducted at a drive ratio of $3 \%$. Thus, the pressure term will be $\delta p / p_{m}=0.03 \cdot e^{i \omega t}$. 
Substituting these values into Equation (17), we will find that the temperature of this interference fringe can oscillate by $\pm 9 \mathrm{~K}$ around its time averaged value of $300 \mathrm{~K}$.

\section{Visualized and reconstructed temperature fields}

\subsection{Grooved channel}

In the grooved channel the flow velocity is described by the Reynolds number defined as $\operatorname{Re}_{\mathrm{g}} \equiv \frac{u_{m} D_{h}}{v}$, where $u_{m}$ is the mean air flow velocity averaged over a cross section at the channel inlet (corresponds to the dimensions of the cross section in the groove region). The critical Reynolds number for the onset of oscillations was found to be approximately $\operatorname{Re}_{g, \text { crit }}$ $=1320$, a flow rate significantly below the value characteristic for the onset of turbulence. The temperature oscillations that mirror the flow structure confirm the existence of a natural frequency in the investigated channel geometry. After the onset of oscillations, significant mixing between groove and bulk flows is initiated and it contributes to heat transfer enhancement. Typical histories of temperature fields corresponding to one period of oscillations at two representative Reynolds numbers, $\operatorname{Re}_{\mathrm{g}}=1580$ and 2890, are shown in Fig. 8.
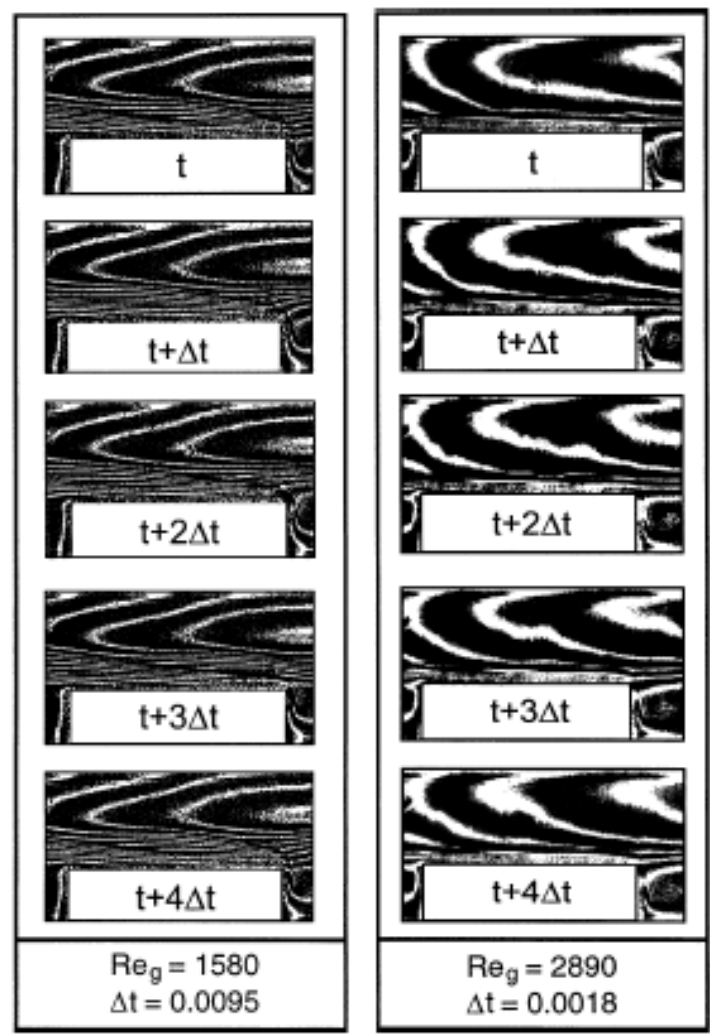

Fig. 8. Temperature fields in the grooved channel during a cycle of self-sustained oscillations at $\operatorname{Re}_{\mathrm{g}}=1580$ and $\operatorname{Re}_{\mathrm{g}}=2890$. 
In the interferometric images shown in Fig. 8 two dominant oscillatory features were observed. The first feature is characterized by traveling waves of different wavelengths and amplitudes in the main channel, visualized by moving isotherms, that are continuously being swept downstream. Typically, for $\operatorname{Re}_{\mathrm{g}}=1580$, the waves are comprised of several isotherms assembled in a stack and these structures are characteristic of the TollmienSchlichting waves in the main channel, which are activated by the Kelvin-Helmholtz instabilities of the free shear layer spanning the groove. The speed of a traveling wave can be determined by measuring the time it takes for the wave peak to traverse a known distance. The frequency of traveling waves is the inverse of the average wave period defined as the time required for two consecutive wave peaks to cross a fixed location during a given time interval. The second characteristic oscillatory feature is observed at a location close to the leading edge of the heated block. The horizontal isotherm separating the main channel and groove regions remains relatively motionless for low Reynolds numbers. As the Reynolds number increases, the isotherm starts "whipping" up and down, which is indicative of vertical velocity components.

For $\operatorname{Re}_{\mathrm{g}}=1580$ the dominant frequency of the oscillatory whip was $29 \mathrm{~Hz}$ and corresponded well to the frequency of the traveling waves in the main channel, $26 \mathrm{~Hz}$. At this Reynolds number two full waves, easily identified in Figure 8 (left), spanned one geometric periodicity length. The data regarding wave characteristics, such as wavelength propagation speed and flow oscillations, obtained for two Reynolds numbers, $\operatorname{Re}_{\mathrm{g}}=1580$ and $\operatorname{Re}_{\mathrm{g}}=2370$, and the number of images evaluated are summarized in Table 1.

\begin{tabular}{|l|c|c|c|c|}
\hline \multicolumn{1}{|c|}{ PARAMETER } & \multicolumn{2}{c|}{$\operatorname{Re}_{g}=1580$} & \multicolumn{2}{c|}{$\operatorname{Re}_{g}=2370$} \\
\hline & Main channel & Groove lip & Main channel & Groove lip \\
\hline Waves/Period, $L / \lambda_{T S}$ & 1.85 & N/A & 2.94 & N/A \\
\hline Mean wave speed $(\mathrm{m} / \mathrm{s})$ & 0.71 & $\mathrm{~N} / \mathrm{A}$ & 1.76 & $\mathrm{~N} / \mathrm{A}$ \\
\hline Dominant frequencies $(\mathrm{Hz})$ & 26 & 29 & 125 & 44,67 \\
\hline Average period (s) & 0.039 & 0.035 & 0.01 & 0.023 \\
\hline No. of periods analyzed & 9 & 6 & 161 & 161 \\
\hline Total images sampled & 350 & 208 & 1582 & 3754 \\
\hline
\end{tabular}

Table 1. Wavelengths, oscillation frequencies and speeds of traveling waves in the grooved channel at $\operatorname{Re}_{\mathrm{g}}=1580$ and $\operatorname{Re}_{\mathrm{g}}=2370$

Increasing the Reynolds number from 1580 to 2370 results in an increase of the frequency of the oscillatory whip and a much more pronounced increase of frequency of the propagating waves. On the average, at $\operatorname{Re}_{g}=2370$, the geometric periodicity contained three TollmienSchlichting waves. These three waves are also present at $\operatorname{Re}_{g}=2890$, but they are not as obvious or easy to recognize in Fig. 8 (right). For $\operatorname{Re}_{g}=2370$, the measured frequencies of the main channel wave activity and oscillating isotherm show more scatter than was the case for the lower Reynolds number. The dominant frequencies indicated in Table 1 have different definitions for $\operatorname{Re}_{g}=1580$ and $\operatorname{Re}_{g}=2370$. For $\operatorname{Re}_{g}=1580$ the flow exhibits a more ordered behavior and the dominant frequency is the average of all the frequencies recorded. The number of image frames that needs to be analyzed to determine frequencies and speeds of these two characteristic features depends primarily on the Reynolds number of the flow 
and on the appropriate recording speed for that particular Reynolds number. For low $\operatorname{Re}_{g}$, when the flow is ordered and well behaved, several hundred images were analyzed. For $\operatorname{Re}_{g}$ greater than 2000, several thousand images were processed sequentially in order to obtain an acceptable sample for quantification of the disorderly flow behavior. These numbers are indicated in Table 1.

From images obtained by holographic interferometry, such as those shown in Fig. 8, temperature fields can be reconstructed using the approach described in Section 4, with the algorithm for situations without the pressure fluctuations. Reconstructed temperature distributions in the basic grooved channel (left) and the grooved channel enhanced with curved vanes near the trailing edge of the heated block (right) for $\operatorname{Re}_{g}=530$ and $\operatorname{Re}_{g}=1580$ are displayed in Fig. 9. From the temperature distributions we can determine temperature gradients near the heated surface, find the values of the local Nusselt numbers along the surface and identify regions with low heat transfer rates and high heat transfer rates for different time instants during an oscillation cycle. Therefore we can analyze both spatial and time dependences of temperature and flow distributions as well as heat transfer. This information allows understanding and quantifying the mechanisms responsible for the heat transfer enhancement and optimizing the geometry of the grooved channel for maximum heat transfer.
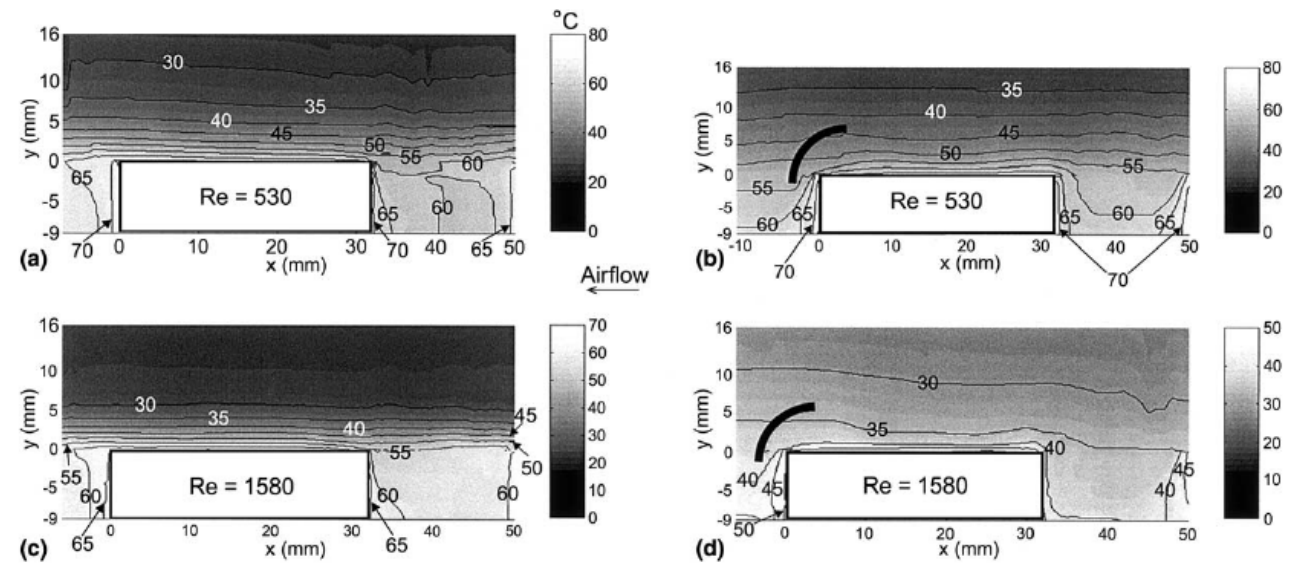

Fig. 9. Temperature fields measured using HI in the basic grooved channel (left) and the grooved channel enhanced with curved vanes near the trailing edge of the heated block (right) for $\operatorname{Re}_{g}=530$ and $\operatorname{Re}_{g}=1580$.

\subsection{Communicating channels}

The Reynolds number in the communicating channels is defined as $\operatorname{Re}_{c} \equiv \frac{3}{2} \frac{u_{m} H}{v}$, where the characteristic length scale $H$ in this definition represents the half-height of the main channel. A temperature distribution obtained by $\mathrm{HI}$ for the steady-state regime is displayed in Fig. 10. The critical Reynolds number for the onset of oscillations was experimentally determined to be around 200 and this result is in good agreement with data obtained numerically (Amon et al. 1992). At Reynolds numbers above the critical value for the onset 
of oscillations the vortices in the communicating region are unsettled and the steady state of the flow is disrupted. The vortex configuration becomes unstable, and vortices are ejected alternately to the top and bottom channels, thus inducing mixing between the vortex and bulk flows.

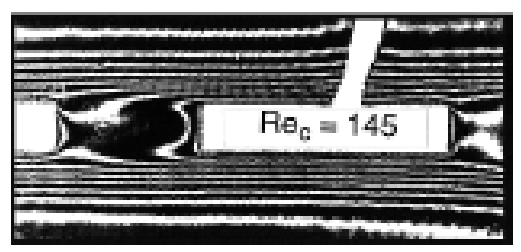

Fig. 10. Instantaneous temperature fields visualized by holographic interferometry in the form of isotherms in the communicating channels at $\operatorname{Re}_{c}=145$, for the steady-state situation.

By analyzing sequences of interferometric images recorded by high-speed camera, different oscillatory regimes and varying oscillatory amplitudes were detected in the communicating channels. Two characteristic flow situations are illustrated schematically in Fig. 11, together with the corresponding temperature fields. One can notice the presence of a) four and b) three traveling waves over the double periodicity length in these images. As the flow structure in the communicating region is in agreement with the traveling wave structure in the main channels, the vortical structures in two successive communicating regions are either a) identical or b) antisymmetric, respectively.

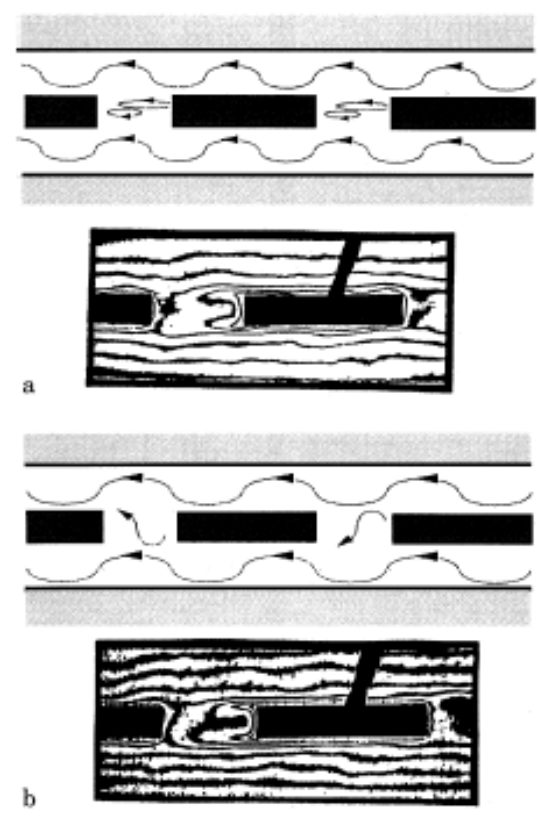

Fig. 11. Oscillations modes in two geometric periodicity lengths for a) four waves and c) three waves in the investigated region. 
The studies on communicating channels led to interesting discoveries regarding flow instabilities: different oscillatory regimes were detected and amplitudes of oscillations varied significantly at the same flow velocity and during the same experimental run. These phenomena, captured by visualizing temperature fields, are illustrated in Figure 12, showing the history of temperature fields during one period of oscillations for the two different oscillatory regimes developing at $\operatorname{Re}_{c}=493$. In the first set of interferometric images displayed in Figure 12 (left), hardly any vortex activity can be observed in the communicating region, indicating reduced lateral mixing. The second sequence of interferometric images in Fig. 12 (right) was recorded during a time interval of the same length as the one corresponding to the sequence shown on the left hand side. The pattern of oscillations has changed, and the set of interferometric images displayed in Fig. 12 (right) shows intensive vortex activity in the communicating region, indicating that vortices ejected into the top and bottom channels improve lateral mixing. The intensity of waviness in the main channels does not change significantly.

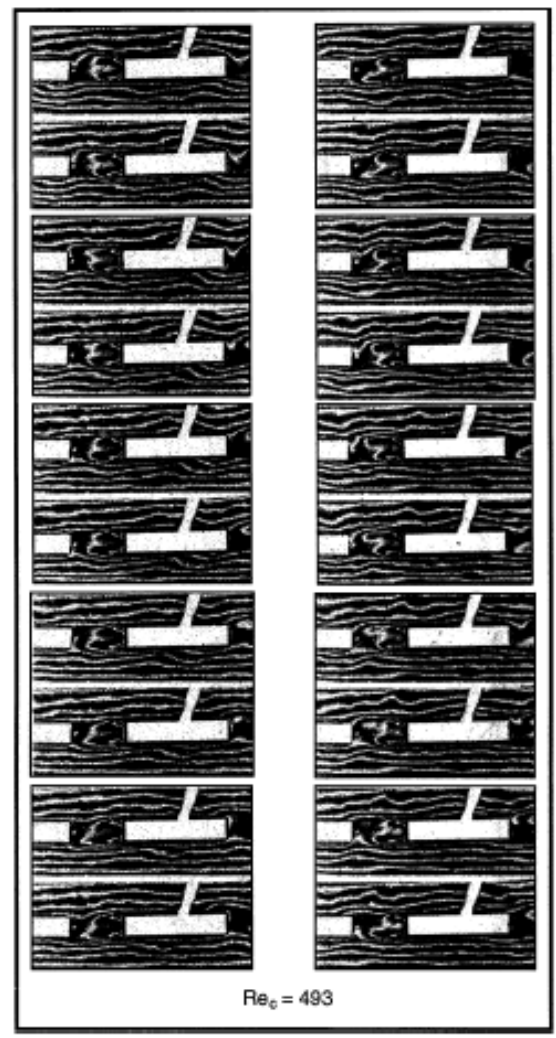

Fig. 12. Time evolution of temperature fields corresponding to two oscillatory regimes visualized by holographic interferometry in the communicating channels at $\operatorname{Re}_{c}=493$.

As discussed in Section 2, in order to demonstrate the advantages of using the infinite fringe field alignment in quantitative flow visualization, in Fig. 3 we compared the characteristic 
features of the fringe patterns developed along the first two heated plates in the communicating channels. These interferometric images were obtained using the finite fringe field alignment (top) and the infinite fringe field alignment (bottom). The initially imposed pattern for the finite fringe field alignment consisted of thin vertical fringes. The temperature distribution obtained by the infinite fringe field alignment allows good qualitative insight into the flow structures in the visualized region, as one can, for example, easily identify the redeveloping thermal and viscous boundary layers along the plates. Wide isotherms develop in the wake downstream of the plate. Fig. 3 clearly demonstrates the advantages of the infinite fringe field alignment in analyzing wave structures in the main channel flow. These cannot be identified in images obtained by the finite fringe field alignment. In order to quantify the oscillatory flow in the vortex region of the communicating channels, we can measure the locations of specific isotherms as a function of time for a sequence of images, such as those in Fig. 12, for a series of Reynolds numbers using digital image processing.

\subsection{Thermoacoustic refrigerator}

The temperature fields in the stack region of the thermoacoustic refrigerator model, visualized by HI, were analyzed and reconstructed quantitatively by first identifying a sequence of interferometric images that describe a complete period of acoustic oscillations in the movie segment of interest. A sequence of 8 interferometric images, representative of one period of acoustic oscillations for a drive ratio of $1 \%$, is presented in Fig. 13.

a)

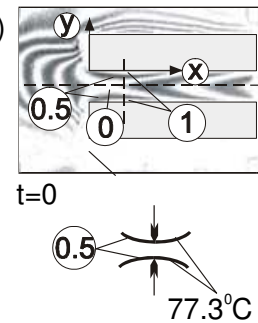

e)

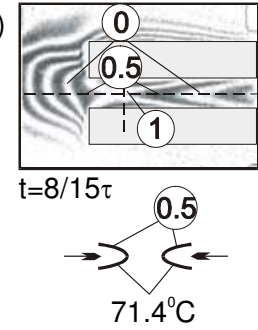

b)

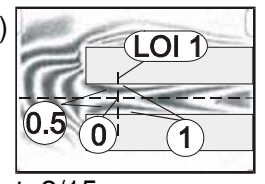

$t=2 / 15 \tau$
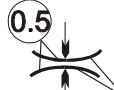

$76.3^{\circ} \mathrm{C}$

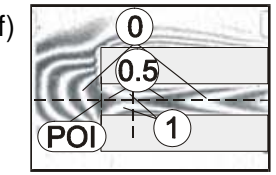

$\mathrm{t}=10 / 15 \tau$

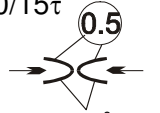

$72.8^{\circ} \mathrm{C}$

pressure antinode c)

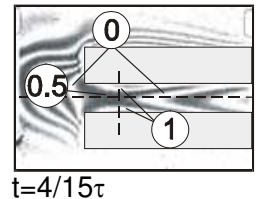

0.5

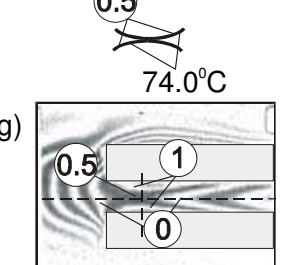

$t=12 / 15 \tau$

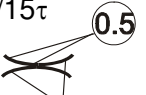

$75.2^{\circ} \mathrm{C}$

pressure node

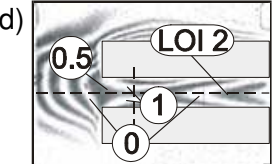

$\mathrm{t}=6 / 15 \tau$
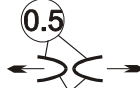

$71.9^{\circ} \mathrm{C}$

h)

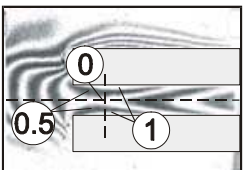

$t=14 / 15 \tau$

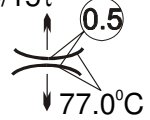

Fig. 13. Oscillating temperature fields around two stack plates of the thermoacoustic refrigerator at a drive ratio $\mathrm{DR}=1 \%$. Images were recorded at a rate of 5,000 picture frames per second. Fringes of the order 0, 0.5 and 1 are indicated in the images and the measured temperature of the fringe of the order of 0.5 is displayed under the image. It should be noted that the temperature of this fringe changes periodically with the change of the acoustic pressure. 
The changes the fringe pattern undergoes during one period of oscillations are analyzed by following the motion of the fringes tagged with the order 0.5 . The changes of temperature of the fringe tagged with interference order 0.5 from $77.3^{\circ} \mathrm{C}$ to $71.4^{\circ} \mathrm{C}$, obtained by taking advantage of the concept of quasi isotherms, are indicated in the schematic for the time instants illustrated in Figure 13.

Prior to the quantitative evaluation, an image specific coordinate system was defined, and image processing as well as curve fit algorithms were applied to determine the spatially and temporally continuous interference order $S(x, y, t)$. Details regarding the image processing procedure are available in the literature (Wetzel, 1998) and were also discussed in Section 4. From the continuous interference order, the time averaged interference order $S_{m}(x, y)$ and the interference order amplitude $S_{A}(x, y)$ were recovered. The latter two quantities were then used to determine the time averaged temperature fields $T_{m}(x, y)$, shown in Fig. 14.

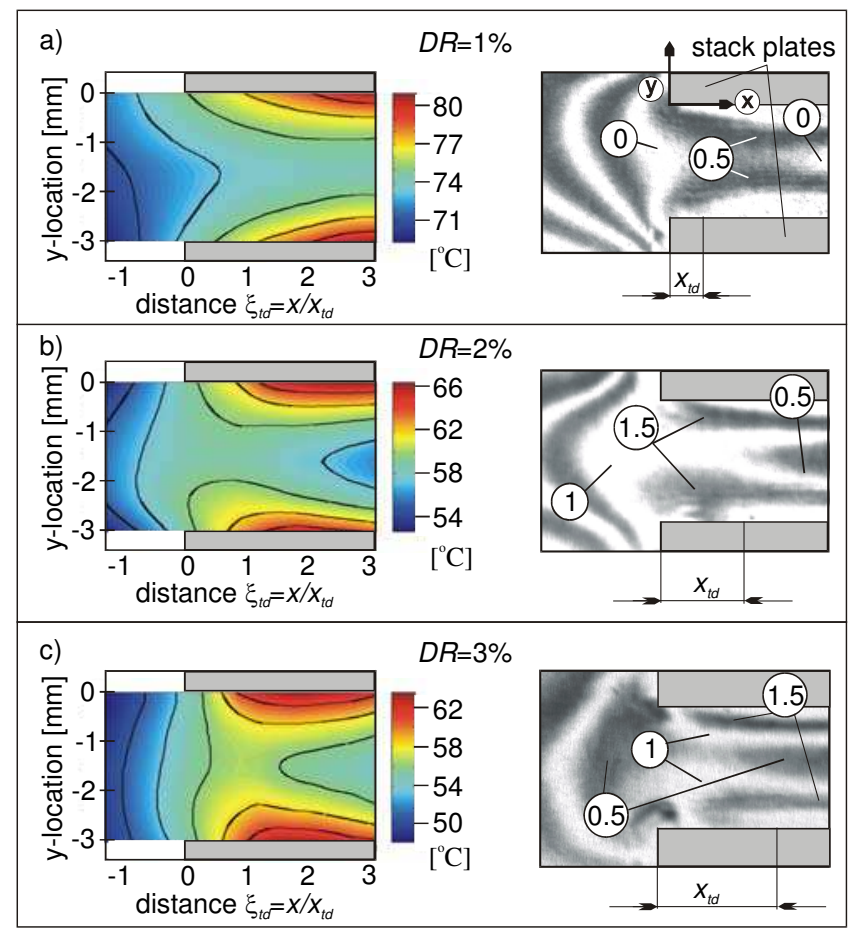

Fig. 14. Time-averaged visualized temperature fields (left: color coded reconstructions from interferometric fringe patterns and right: corresponding interferometric fringe patterns) at the hot end of the two stack plates for drive ratios DR of $1 \%, 2 \%$ and $3 \%$.

An examination of the time averaged temperature field and interferometric image in Figure 14a shows that for the drive ratio $D R=1 \%$ the working fluid along the axis of the channel is colder than at the stack plates. This trend is confirmed by considering the interferometric image on the right hand side of Figure 14a. This image demonstrates that the colder fringe of order 0 bends over the warmer fringe of order 0.5 . Such a temperature distribution and fringe pattern indicate that heat is being transferred from the stack plates to the working 
fluid. This behavior is expected, since heat is generated in the stack plates by resistive heaters in addition to the thermoacoustic heating.

The shape of the isotherms in Figures $14 \mathrm{~b}$ and $c$, representing the time averaged temperature fields for the drive ratios $D R=2 \%$ and $3 \%$ becomes more complicated than for $D R=1 \%$, with the shape of the isotherms reflecting vortex shedding in the region $0 \leq \xi \leq 1$, the edge of the stack plate. The temperature of the working fluid is higher than that of the lower stack plate close to the edge of the plate $(\xi \sim 0)$. Both fringe pattern and temperature distribution indicate that heat is transferred into the lower stack plate at $\xi \sim 0$.

Time dependent temperature distributions $T(x, y, t)$ in the upper half of the channel between the two investigated stack plates for the drive ratio $D R=3 \%$ are shown in Figure 15 .

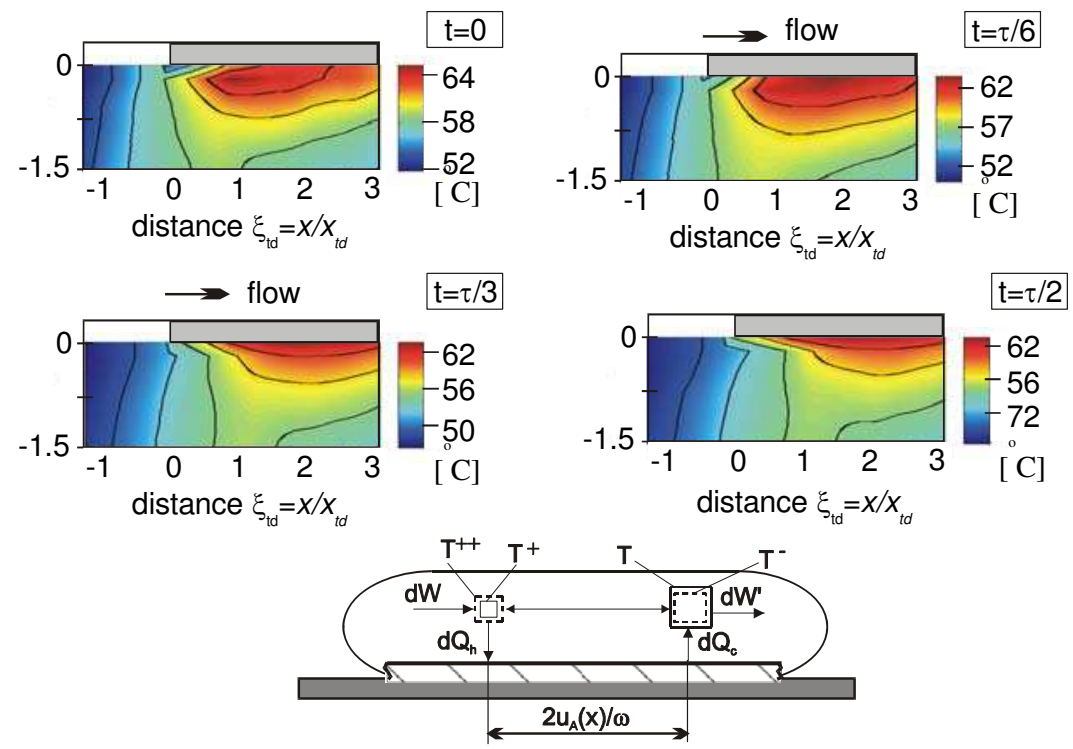

Fig. 15. Visualization of the thermoacoustic effect through the reconstructed temperature distributions during one half of the acoustic cycle. The first image illustrates full compression and the last one full expansion with the associated reversal of the direction of the heat flow, which is the characteristic of the thermoacoustic effect.

The flow direction is also indicated in Figure 15, and the length of the arrow is proportional to the flow velocity. The instanteneous temperature distributions correspond to four time instants within one half of the acoustic cycle, $t=0, \tau / 6, \tau / 3$ and $\tau / 2$, steps 2 through 4 , as described by the gas parcel model (Swift, 1988). At $\mathrm{t}=0$ (top left) the working fluid is fully compressed and hotter than the stack plate in the region $0<\xi<3$. This is indicative of heat being transferred into the stack plate. This time instant therefore corresponds to the second step in the gas parcel model with the working fluid at the temperature $T^{++}$(Figure 6). As time progresses, at $t=\tau / 6$ and $\tau / 3$ (top right and bottom left), the working fluid gradually expands and cools down to the temperature $T^{-}$. This time sequence corresponds to the third step in the gas parcel model. The fourth step in the gas parcel model is the last temperature field image (bottom right) in Figure 15. At this time instant the working fluid is 
fully expanded, it is colder than the stack plate, and heat is being transferred from the stack plate to the working fluid, illustrated as $d Q_{c}$. During the second half of the cycle the working fluid is being compressed, and the temperature distribution goes through the phases displayed in Figure 15 in reversed order. The temperature gradient changes sign and therefore heat is being transferred from the stack plate into the working fluid at $\xi=0$.

\section{Conclusions}

The results reported in this paper demonstrate that HI, apart from the measurement of unsteady temperature distributions and local heat transfer, also allows the quantitative study of flow characteristics for certain classes of oscillatory flows coupled with heat transfer, and it can be recommended in situations when quantitative local velocity data are not required. We can conclude that through visualization of temperature fields we can indirectly study flow instabilities and resonant modes, as well as measure wavelength, propagation speed and frequency of traveling waves. HI allows the visual identification of separated flow regions, and provides limited amount of detail regarding the recirculation region. However, it should also be pointed out that the method does not replace the widely accepted, nonintrusive and quantitative velocity measurement techniques, such as LDA or PIV, rather it complements them.

The advantage of the approach introduced in the paper over "classical" visualization methods (such as the use of tracers that allows qualitative insight only) is that quantitative information on the structure of flow and temperature fields as well as heat transfer is obtained simultaneously, using the same experimental setup and during the same experimental run, thus yielding consistent flow and heat transfer data. This feature makes the technique particularly attractive for applications such as the development of flow control strategies leading to heat transfer enhancement. Using temperature as tracer offers the additional advantage that a quantitative analysis of high-speed thermofluid processes becomes possible, since optical measurement techniques are nonintrusive, and they, due to the high speed of light, have virtually no inertia. In the study of complex flow situations and flow instabilities, the investigated process can be very sensitive to disturbances, and the injection of dye or tracer particles can interfere with the flow, causing it to switch to another oscillatory regime due to perturbations. With $\mathrm{HI}$, the contamination of the test section by tracers is avoided, thus allowing longer experimental runs and analyses of different steady states and transients during a single experimental run.

The effort involved in the study of high-speed, unsteady flows, in the measurement of phase shift and the extraction of quantitative information from the obtained images, is significant. When analyzing oscillatory flows, the required recording frequencies are usually at least one order of magnitude higher than the frequency of the physical process. Due to the high sampling rates, thousands of images are generated within seconds, and these images are then successively processed to extract the required quantitative information. Algorithms for a completely automated analysis of images recorded by holography, PIV and HPIV are available. Algorithms that allow an automated analysis of images generated by $\mathrm{HI}$ have been described in the literature, however, at this point of time the issue has not yet been adequately resolved. This is especially true for unsteady processes. Therefore the quantitative evaluation of interferometric fringe patterns still poses a challenge and limits the applications of HI. 


\section{Acknowledgments}

The research reported in the paper was supported by the National Science Foundation and the Office of Naval Research. Dr. Martin Wetzel and Dr. Eric Kang were instrumental in conducting the experiments as well as in the data analyis.

\section{References}

Amon, C. H.; Majumdar, D.; Herman, C. V.; Mayinger, F.; Mikic, B. B.; Sekulic, D. P. (1992) Numerical and experimental studies of self-sustained oscillatory flows in communicating channels, Int. J. Heat and Mass Transfer, Vol. 35, 3115-3129

Cooper, W. L.; Yang, K. T.; Nee, V. W. (1993) Fluid mechanics of oscillatory and modulated flows and associated applications in heat and mass transfer - A review, J. of Energy, Heat and Mass Transfer, Vol. 15, 1-19

Farhanieh, B.; Herman, C.; Sunden, B. (1993) Numerical and experimental analysis of laminar fluid flow and forced convection heat transfer in a grooved duct, Int. J. Heat and Mass Transfer, Vol. 36, No. 6, 1609-1617

Ghaddar, N. K.; Korczak, K. Z.; Mikic, B. B.; Patera, A. T. (1986a) Numerical investigation of incompressible flow in grooved channels. Part 1. Stability and self-sustained oscillations, J. Fluid Mech., Vol. 163, 99-127

Ghaddar, N. K.; Magen, M.; Mikic, B. B.; Patera, A. T. (1986b) Numerical investigation of incompressible flow in grooved channels. Part 2. Resonance and oscillatory heat transfer enhancement, J. Fluid Mech., Vol. 168, 541-567

Greiner, M.; Chen, R.-F.; Wirtz, R. A. (1990) Heat transfer augmentation through wall-shapeinduced flow destabilization, ASME Journal of Heat Transfer, Vol. 112, May 1990, 336341

Greiner, M. (1991) An experimental investigation of resonant heat transfer enhancement in grooved channels, Int. J. Heat Mass Transfer, Vol. 34, No. 6, 1383-1391

Hauf, W.; Grigull, U. (1970) Optical methods in heat transfer, in Advances in Heat Transfer, Vol. 6, Academic Press, New York

Herman, C., 2000, The impact of flow oscillations on convective heat transfer, Annual Review of Heat Transfer, Editor: C.-L. Tien, Vol. XI, Chapter 8, pp. 495-562, invited contribution.

Kang, E. (2002) Experimental investigation of heat transfer enhancement in a grooved channel, Dissertation, Johns Hopkins University, Baltimore, MD, USA.

Karniadakis, G. E.; Mikic, B. B.; Patera, A. T. (1987), Heat transfer enhancement by flow destabilization: application to the cooling of chips, Proc. Int. Symposium on Cooling Technology for Electronic Equipment, 587-610

Mayinger, F. Editor (1994) Optical measurements - Techniques and applications, Springer Verlag, Berlin

Patera, A. T.; Mikic, B. B. (1986), Exploiting hydrodynamic instabilities. resonant heat transfer enhancement, Int. J. Heat and Mass Transfer, Vol. 29, No. 8, 1127-1138

Panknin, W., 1977, Eine holographische Zweiwellenlangen-Interferometrie zur Mesung uberlagerter Temperatur- und Konzentrationsgrenzschichten, Dissertation, University of Hannover, Germany.

Swift, G.W. (1988) Thermoacoustic Engines, J. Acoust. Soc. Am. 84(4), Oct., 1145-1180

Vest, C. M. (1979) Holographic interferometry, John Wiley \& Sons, New York 
Wetzel, M. (1998) Experimental investigation of a single plate thermoacoustic refrigerator, Dissertation, Johns Hopkins University, Baltimore, MD, USA.

Wheatley, J.C.; Hofler, T.; Swift, G.W.; Migliori, A. (1983) An intrinsically irreversible thermoacoustic heat engine, J. Acoust. Soc. Am., 74 (1), July, 153-170 


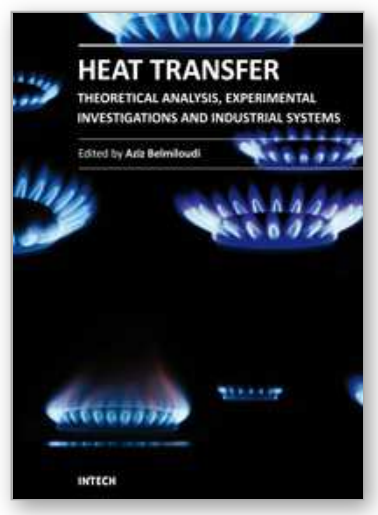

\author{
Heat Transfer - Theoretical Analysis, Experimental Investigations \\ and Industrial Systems \\ Edited by Prof. Aziz Belmiloudi
}

ISBN 978-953-307-226-5

Hard cover, 654 pages

Publisher InTech

Published online 28, January, 2011

Published in print edition January, 2011

Over the past few decades there has been a prolific increase in research and development in area of heat transfer, heat exchangers and their associated technologies. This book is a collection of current research in the above mentioned areas and discusses experimental, theoretical and calculation approaches and industrial utilizations with modern ideas and methods to study heat transfer for single and multiphase systems. The topics considered include various basic concepts of heat transfer, the fundamental modes of heat transfer (namely conduction, convection and radiation), thermophysical properties, condensation, boiling, freezing, innovative experiments, measurement analysis, theoretical models and simulations, with many real-world problems and important modern applications. The book is divided in four sections : "Heat Transfer in Micro Systems", "Boiling, Freezing and Condensation Heat Transfer", "Heat Transfer and its Assessment", "Heat Transfer Calculations", and each section discusses a wide variety of techniques, methods and applications in accordance with the subjects. The combination of theoretical and experimental investigations with many important practical applications of current interest will make this book of interest to researchers, scientists, engineers and graduate students, who make use of experimental and theoretical investigations, assessment and enhancement techniques in this multidisciplinary field as well as to researchers in mathematical modelling, computer simulations and information sciences, who make use of experimental and theoretical investigations as a means of critical assessment of models and results derived from advanced numerical simulations and improvement of the developed models and numerical methods.

\title{
How to reference
}

In order to correctly reference this scholarly work, feel free to copy and paste the following:

Cila Herman (2011). Quantitative Visualization of Heat Transfer in Oscillatory and Pulsatile Flows, Heat Transfer - Theoretical Analysis, Experimental Investigations and Industrial Systems, Prof. Aziz Belmiloudi (Ed.), ISBN: 978-953-307-226-5, InTech, Available from: http://www.intechopen.com/books/heat-transfer-theoreticalanalysis-experimental-investigations-and-industrial-systems/quantitative-visualization-of-heat-transfer-inoscillatory-and-pulsatile-flows

\section{INTECH}

open science | open minds

\author{
InTech Europe \\ University Campus STeP Ri \\ Slavka Krautzeka 83/A
}

\author{
InTech China \\ Unit 405, Office Block, Hotel Equatorial Shanghai \\ No.65, Yan An Road (West), Shanghai, 200040, China
}


51000 Rijeka, Croatia

Phone: +385 (51) 770447

Fax: +385 (51) 686166

www.intechopen.com
中国上海市延安西路65号上海国际贵都大饭店办公楼 405 单元 Phone: +86-21-62489820

Fax: +86-21-62489821 
(C) 2011 The Author(s). Licensee IntechOpen. This chapter is distributed under the terms of the Creative Commons Attribution-NonCommercialShareAlike-3.0 License, which permits use, distribution and reproduction for non-commercial purposes, provided the original is properly cited and derivative works building on this content are distributed under the same license. 